\title{
Erratum: Gravitational collapse of rotating supermassive stars including nuclear burning effects [Phys. Rev. D 96, 083016 (2017)]
}

\author{
Haruki Uchida, Masaru Shibata, Takashi Yoshida, Yuichiro Sekiguchi, and Hideyuki Umeda
}

(Received 28 November 2018; published 14 December 2018)

\section{DOI: 10.1103/PhysRevD.98.129901}

We found that the numerical code used in our paper [1] has some errors in the part of calculating the effect of nuclear burning. Then, we performed simulations for models A1-A4 again with the modified code and found that the errors made little change in the main results and conclusions of our paper.

However, we found that this error changes the conclusion of Appendix A. In Appendix A, we compared the results of two simulations with the same initial conditions but with different formalisms to incorporate the effects of nuclear burning. One is the formalism described as

$$
\nabla_{\mu} T^{\mu \nu}=\frac{\rho u^{\nu}}{c} \dot{q}
$$

where $T_{\mu \nu}, \rho, u^{\nu}, c$, and $\dot{q}$ are the energy-momentum tensor, rest-mass density, 4-velocity of the fluid, speed of light, and energy generation rate of the nuclear burning, respectively. Here, we considered CNO cycle and triple-alpha reactions (model T1). The other is that described in Sec. II D (model T2). Then, we found that when we employed an initial condition with large metallicity $\left(Z_{\mathrm{CNO}} \gtrsim 10^{-3}\right)$ the results of the two simulations did not agree with each other. We suspected that this difference would be due to the fact that Eq. (1.1) violates the energy-momentum conservation and this violation would be accumulated and finally induce the unnatural results for model $\mathrm{T} 1$.

However, we simulated these models again by using the modified code and found that the numerical results of these simulations agree well each other. Figure 1 here shows the modified version of Fig. 23. A black hole is formed for all the models, and $Q /\left|E_{\text {ini }}\right|$ agrees well in models T1 and T2. Here, $Q$ and $E_{\text {ini }}$ are the released rest-mass energy due to nuclear burning and the initial total energy, respectively. Thus, we withdraw the above argument and conclude that the formulation described in Eq. (1.1) does not lead to such unnatural results. We apologize if a reader has any inconvenience from our incorrect result.

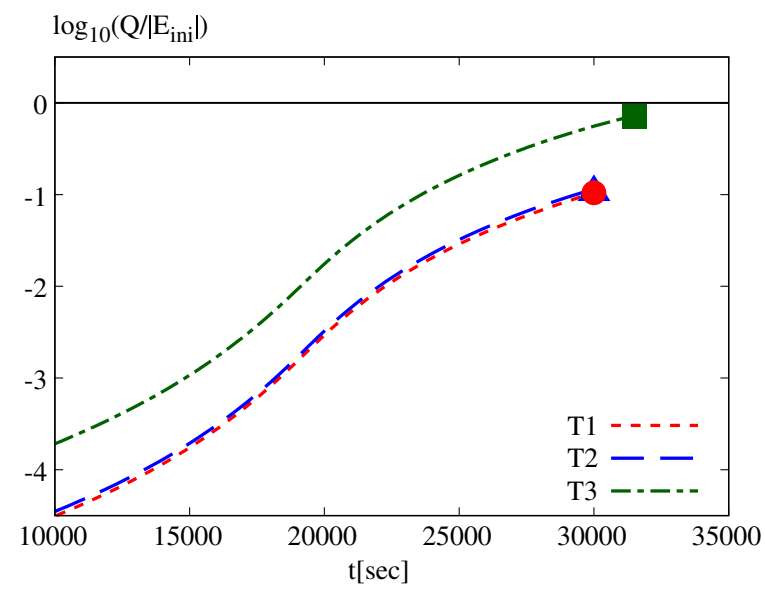

FIG. 1. Time evolution of the ratio of the released rest-mass energy due to nuclear burning, $Q$, to the initial total energy, $E_{\text {ini }}$, with the modified numerical code. $Q$ and $E_{\text {ini }}$ are defined in Eqs. (A3) and (A4), respectively. The dotted, dashed, and dashed-dotted curves denote for models T1, T2, and T3, respectively. For models T1 and T2, a black hole is formed at $t \approx 30,000 \mathrm{~s}$ (filled triangle and circle), and for model $\mathrm{T} 3$, a black hole is formed at $t \approx 31,500 \mathrm{~s}$ (filled square).

[1] H. Uchida, M. Shibata, T. Yoshida, Y. Sekiguchi, and H. Umeda, Phys. Rev. D 96, 083016 (2017). 